\title{
Transcatheter Decompression of the Left Atrium in an Adolescent with a Double Inlet Left Ventricle, Severe Parachute Mitral Valve Stenosis and Pulmonary Hypertension
}

\author{
Y. H. Cai, Q. Li, Z. Li, G. Yang, D. Schranz* \\ MINGCI Cardiovascular Hospital Heart Center, Wuxi, China \\ Email: *dietmar.schranz@paediat.med.uni-giessen.de
}

How to cite this paper: Cai, Y.H., Li, Q., Li, Z., Yang, G. and Schranz, D. (2017) Transcatheter Decompression of the Left Atrium in an Adolescent with a Double Inlet Left Ventricle, Severe Parachute Mitral Valve Stenosis and Pulmonary Hypertension. World Journal of Cardiovascular Diseases, 7, 451-457.

https://doi.org/10.4236/wjcd.2017.712044

Received: September 19, 2017

Accepted: December 11, 2017

Published: December 14, 2017

Copyright $(\odot) 2017$ by authors and Scientific Research Publishing Inc. This work is licensed under the Creative Commons Attribution International License (CC BY 4.0).

http://creativecommons.org/licenses/by/4.0/

\begin{abstract}
A 17-year-old adolescent with non-operated double inlet left-ventricle and severe stenosed parachute mitral valve is reported. He was admitted with repetitive syncope related to intermittent atrial fibrillation. Life-threatening syncope combined with pre- and post-capillary pulmonary hypertension together with his single ventricle pathophysiology led to the decision for left atrial decompression by percutaneous static atrial septum ballooning after transseptal needle perforation. Aiming to create a restrictive atrial septum defect, unloading of the left atrium without disturbing the balanced hemodynamics was directed for a long-term palliation or as a basis for a further surgical follow-up approach.
\end{abstract}

\section{Keywords}

Double Inlet Left Ventricle, Parachute Mitral Valve, Pulmonary Hypertension, Left Atrial Decompression

\section{Introduction}

Double inlet left ventricle (DILV) belongs to the family of univentricular hearts (UVH). Multiple morphological variants can be associated, but mitral valve dysfunction is uncommon [1]. The prognosis of UVH is usually poor without early surgical interventions; a median survival of 14 years was reported [2]. Only the morphological variant, in which the pulmonary blood flow $(\mathrm{Qp})$ is limited by an right ventricular outflow tract obstruction or a significant pulmonary hypertension, might have the chance for a natural long-term course into adulthood. It 
is presupposed that, a balanced Qp to systemic blood flow (Qs) is achieved avoiding heart-lung congestion on the one or deep cyanosis on the other hand [3]. We report a 17-year-old young man with DILV, severe stenosis of a parachute mitral valve and pulmonary hypertension. He was admitted with dyspnea and syncope related to intermittent atrial fibrillation. Concomitant to medical treatment, left atrial decompression was performed by percutaneous transcatheter approach achieving a restrictive atrial communication as a basis for long-term or further surgical master plan.

\section{Case Report}

A 17-year-old young man with a univentricular physiology admitted Mingci Cardiovascular Center, Wuxi with complaints of dyspnea and syncope associated to intermittent atrial fibrillation. No surgery treatment was performed before. He was referred for surgery of the mitral valve stenosis. The patient's intellectual and somatic development was normal; his body weight and length was $70 \mathrm{~kg}$ and $182 \mathrm{~cm}$, respectively. At rest, the breath rate was normal and only discrete cyanotic clinical signs were recognizable (pulseoximetry oxygen saturation, $\left.\mathrm{SaO}_{2} 90 \%-93 \%\right)$. Also after admission, syncope's became obvious correlated to intermittent atrial fibrillations; two short-time resuscitations were necessary. At our institution the exact morphological diagnosis was made by echocardiography (ECHO) and cardiac computer tomography (cCT). A d-loop cardiac anatomy of a DILV with intact atrial septum combined with a high degree stenosis of a parachute mitral valve became obvious (Figure 1); additionally, a moderate regurgitation of the tricuspid valve was observed. From Doppler-Echo examination as well as CCT data a discrete obstructive sub-pulmonary region was assumed despite of an unrestrictive ventricular septum defect communicating with a hypoplastic right ventricle (Figure 2). The invasive hemodynamic evaluation did not reveal a relevant systolic gradient across the right ventricular outflow tract; the measured pulmonary artery pressure was $94 / 54 \mathrm{mmHg}$, the corresponding systemic artery pressure $96 / 73 \mathrm{mmHg}$. The diastolic pressure ratio was

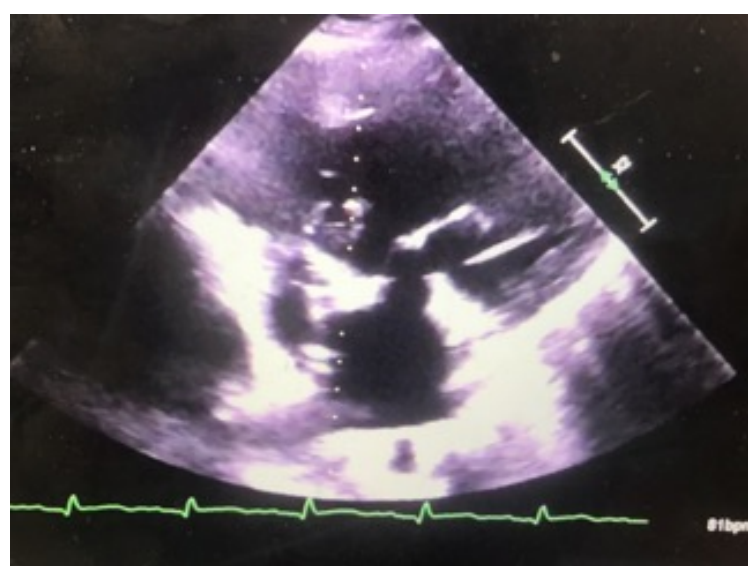

Figure 1. Transthoracic echocardiography shows by apical view a double inlet left ventricle with intact atrial septum and a stenosed parachute mitral valve. 


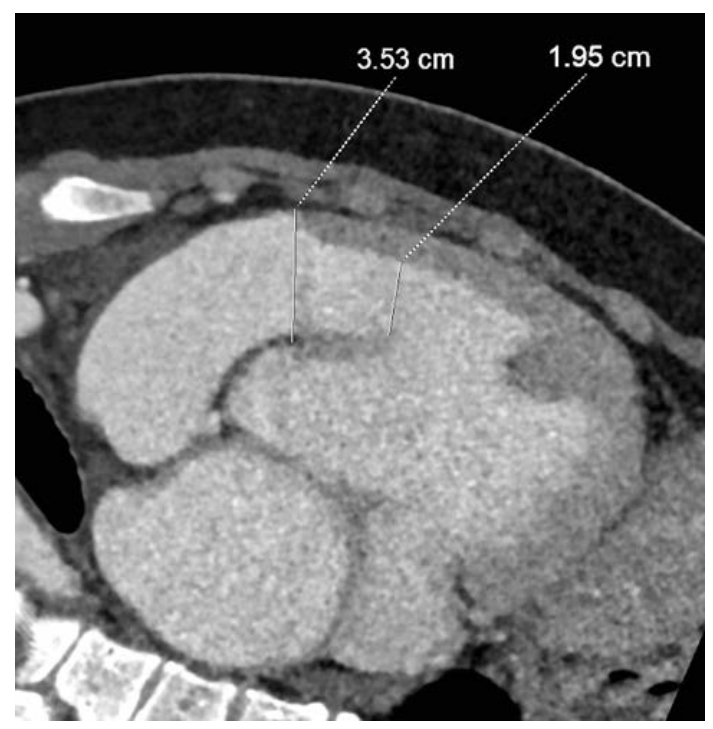

Figure 2. Oblique Cardio Computer tomography (cCT) shows an unrestrictive ventricle septum defect communicating with a hypoplastic right ventricle and slightly reduced right ventricular outflow tract $(19 \mathrm{~mm})$ and an enlarged pulmonary artery $(35 \mathrm{~mm})$.

0.74 . The pulmonary artery oxygen saturation measured by co-oximetric analysis was $84 \%\left(\mathrm{SaO}_{2} 90 \%\right)$ the corresponding systemic vein $\mathrm{SO}_{2} 64 \%$. The approximated pulmonary to systemic blood flow (Qp:Qs) ratio was calculated as 1.7. The systolic UVH-function, qualitatively analyzed by ECHO revealed sufficient; the invasively measured end-diastolic pressure of the dominant left ventricle corresponded with the mean right atrial pressure of $8 \mathrm{mmHg}$. Considering the CW-Doppler based calculated mean pressure gradient of $20-25 \mathrm{mmHg}$ across the mitral valve (MV) with an opening area of $1.52 \mathrm{qcm}$, allowed to assume a mean left atrial pressure (LAP) between $25-30 \mathrm{mmHg}$. Therefore, a combined pulmonary hypertension was diagnosed with a diastolic pressure gradient of about $25-30 \mathrm{mmHg}$. In addition to medical, in particular antiarrhythmic drug treatment, the decision for creation of a restrictive atrial septum defect was made for partial decompression of the left atrium. Briefly, under exclusive local anesthesia a goal-directed short intervention was performed to create a defined, but still restrictive atrial communication. Fluoroscopy guided Brockenbrough technique was performed utilizing a St. Jude Medical ${ }^{\circledR} 8 \mathrm{Fr}$ trans-septal needle system. After uneventful trans-septal puncture and advancing the $8 \mathrm{~F}$ sheath to the LA, a mean LAP of $26 \mathrm{mmHg}$ was measured corresponding with a trans-septal pressure gradient of $18 \mathrm{mmHg}$; subsequent to the diagnostic measures static dilatation of the atrial septum was performed utilizing a Atlas $\left(\right.$ Bard $\left.^{\circledR}\right)$ high pressure balloon (width $24 \mathrm{~mm}$, length $40 \mathrm{~mm}$ ). The balloon was several times inflated by a maximal pressure of $14 \mathrm{~atm}$. Based on our experience with static ballooning of the atrial septum for heart failure therapy [4], utilizing a $24 \mathrm{~mm}$ highpressure balloon should allow to create a sufficient, but still restrictive atrial septum defect (rASD) of maximal $10-12 \mathrm{~mm}$ diameter. The mean LAP decreased to $16 \mathrm{mmHg}$, and the pressure gradient between both, the right and left 
atrium to $8 \mathrm{mmHg}$; the right atrial pressure (RAP) as well as the TV regurgitation did not increased by the induced left-to-right shunt (Figure 3 ). The short follow-up ECHO data, 10 days later just before the patient was discharged home showed an inspiration related collapsing inferior caval vein despite an unchanged but still moderate TV regurgitation. The mean CW-Doppler pressure gradient across the atrial septum as well as mitral valve was decreased to about $11 \mathrm{mmHg}$; control cCT confirmed the shrinking left atrial size; the measured diameter of the right pulmonary vein was decreased from $22.5 \mathrm{~mm}$ to $17 \mathrm{~mm}$ eight days after LA-decompression (Figure 4(a), Figure 4(b)). The maximal CW-Doppler velocity across the RVOT increased corresponding to a calculated gradient to $50 \mathrm{mmHg}$; the pulsoximetric measured oxygen saturation at rest ranged now between $93 \%$ and $95 \%$. Currently, almost 3 months after the interventional approach no episodes of AF occurred by a concomitant medical therapy consisting of a metoprolol $2 \times 47.5 \mathrm{mg}$, spironolactone $1 \times 25 \mathrm{mg}$, acetyl-salicylate acid (ASS) $1 \times 100 \mathrm{mg}$, enalapril $1 \times 5 \mathrm{mg}+2.5 \mathrm{mg}$ and bosen$\tan 1 \times 125 \mathrm{mg}+1 \times 67.5 \mathrm{mg}$; previously performed amiodarone and intermittent oxygen treatment was stopped.

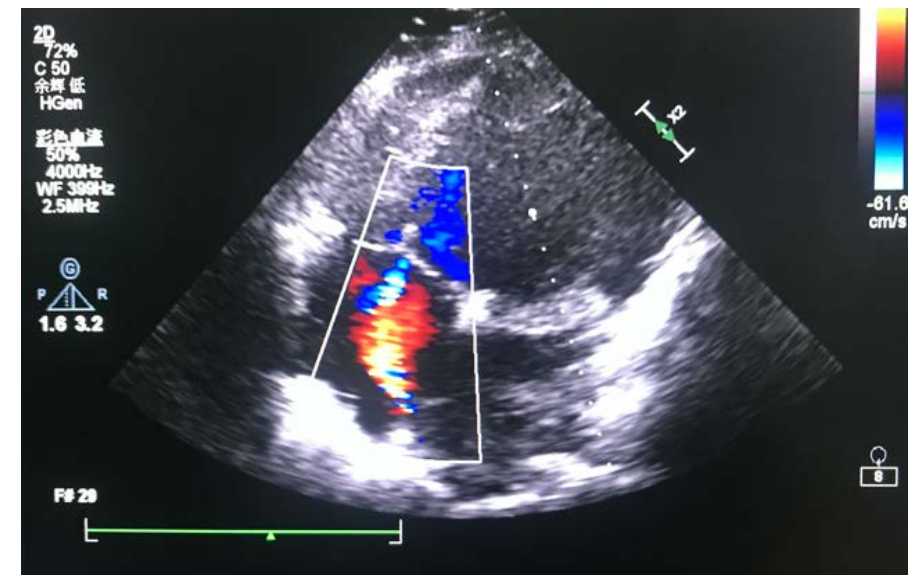

Figure 3. Demonstrates the color-Doppler left-to-right shunt across a created restrictive atrial septum defect (rASD); here during the ventricular systole an additional discrete tricuspid valve regurgitation is shown.

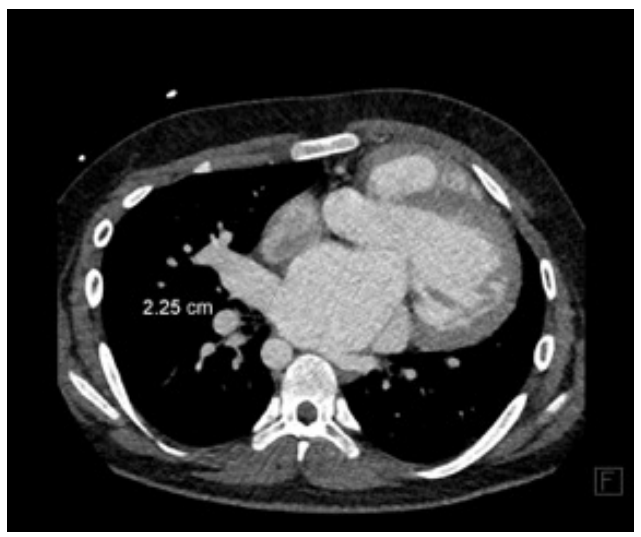

(a) 


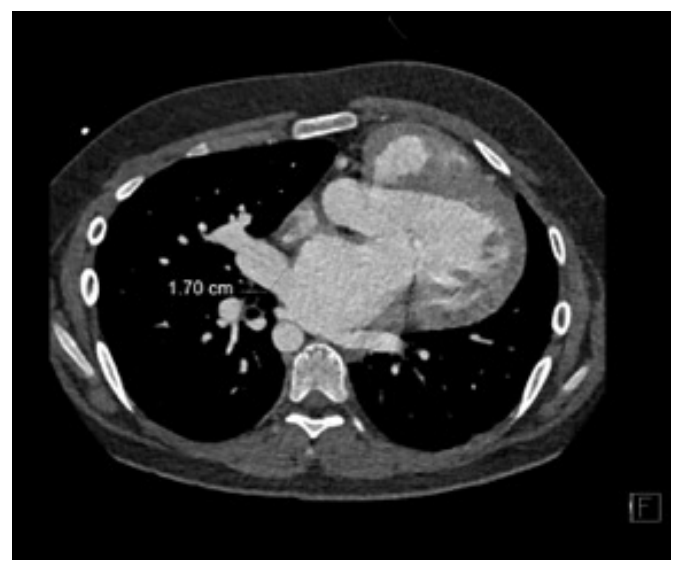

(b)

Figure 4. Shows the left atrium by cCT imaging: (a) before the creation of a rASD a right pulmonary vein (rPV) of $22.5 \mathrm{~mm}$ is measured; (b) with rASD the rPV diameter decreased to $17 \mathrm{~mm}$.

\section{Discussion}

To our knowledge, the described percutaneous intervention is unique in context of such a complex anatomical entity. Parachute valve stenosis associated with a DILV is a rare entity [1] [5]. The reported a young patient admitted Mingci Hospital without any surgical approach in his history. The balanced hemodynamics of his left-type UVH guaranteed a normal somatic development and only minimal exercise intolerance. The admission was urged on episodes of atrial fibrillation with in part severe hemodynamic consequences. Considering the morphological and hemodynamic data, following questions raised and were discussed in the team. Is the precapillary component of the PH fixed because of an "unrestrictive" pulmonary artery blood flow since he was born or was the mitral valve obstruction protective by avoiding congestive heart failure and guaranteeing normal somatic growth-up by achieving a $\mathrm{PH}$ with an adequate blood passage? Did the LAP increased over time? Was the gradually increased LAP together with its related LA wall remodeling and pulmonary vein (PV) distention (right PV of $22 \mathrm{~mm}$ by $\mathrm{cCT}$ ) responsible for the acquired atrial fibrillation? Based on the clinical performance, morphological and hemodynamic data as well as discussed questions following therapeutic strategies were considered: a) high-risk open-heart surgery for replacement of the mitral valve; the surgical approach should be performed under the hypothesis that the patient's combined $\mathrm{PH}$ and arrhythmia would be significantly influenced. At the best, the patient would additionally need a pulmonary banding, if the post-capillary component were abolished. b) Two also discussed procedures were declined: ballooning of congenitally stenosed, but already slightly insufficient parachute mitral valve as well as a transapical valve implantation (TAVI) because of missing special valve materials. c) Step-by-step interventional-surgical hybrid approach starting with creation of a restrictive atrial communication followed by a minor or perhaps no further surgical approach. It was hypothesized, that achieving a second left atrial 
outlet would decompress the left atrial with a significant impact on the incidence of atrial arrhythmias combined with re-balancing the beneficial hemodynamics by which the patient developed very well with only minimal exercise intolerance. However, complete unloading of the left atrium by creation an unrestricted ASD was not aimed in terms of volume overloading of the already moderate insufficient TV, and of its non-calculable effects on the intra-cardiac shunt conditions despite its functional univentricular heart. Considering the risk-benefit ratio, we decided for plan $\mathrm{C}$ at the first and like demonstrated as a low-risk, but efficient, therapeutic step. The immediate results of a controlled LA unloading were convincing. Clinical condition improved and so far no relapse of arrhythmic episodes was observed by concomitant, but well tolerable medical therapy without amiodarone as it became necessary at admission. The additional drug therapy was directed to improve the Qp:Qs-balance favoring the systemic blood flow $\left(\mathrm{SaO}_{2}\right.$ increased to $\left.93 \%-95 \%\right)$ and to influence the pulmonary vascular resistance by bosentan considering a decreased left atrial pressure to an upper normal range. The increased velocity corresponding to CW-Doppler gradient of 50 $\mathrm{mmHg}$ across the RVOT already a couple of days after the intervention were already promising. In case of a further uneventful clinical course, an invasive re-evaluation is provided after a six months interval. Expecting a still open, but restrictive ASD together with a further increase of the RVOT-obstruction; alternatively, if invasively assessed again and hemodynamics should show proof, a pulmonary banding is considered.

\section{Conclusion}

In conclusion, decompression of a left atrium by creating a restrictive communication is a very cost-effective therapeutic strategy, if symptoms of pulmonary congestion or intrinsic left atrial problems as atrial tachycardia have become obvious. Considering the safety and effectiveness of static ballooning for creating a restrictive ASD, the indication of such an interventional procedure should be expanded and pathophysiologically driven and not by the availability of any devices to regulate inter-atrial flows, which are just lunched on the market [6].

\section{References}

[1] Bevilacqua, M., Sanders, S.P., Van Praagh, S., Colan, S.D. and Parness, I. (1991) Double-Inlet Single Left Ventricle: Echocardiographic Anatomy with Emphasis on the Morphology of the Atrio-Ventricular Valves and Ventricular Septal Defect. Journal of the American College of Cardiology, 18, 559-568. https://doi.org/10.1016/0735-1097(91)90615-G

[2] Hager, A., Kaemmerer, H., Eicken, A., Fratz, S. and Hess, J. (2002) Long-Term Survival of Patients with Univentricular Heart Not Treated Surgically. The Journal of Thoracic and Cardiovascular Surgery, 123, 1214-1217. https://doi.org/10.1067/mtc.2002.122535

[3] Park, S.J., Kwak, C.H. and Hwang, J.Y. (2007) Long-Term Survival in Double Inlet Left Ventricle Combined with Pulmonary Stenosis and Parachute Mitral Valve. A Rare Case. International Heart Journal, 48, 261-267. 
https://doi.org/10.1536/ihj.48.261

[4] Schranz, D., Akintuerk, H. and Voelkel, N.F. '(2017) End-Stage' Heart Failure Therapy: Potential Lessons from Congenital Heart Disease: From Pulmonary Artery Banding and Interatrial Communication to Parallel Circulation. Heart, 103, 262-267. https://doi.org/10.1136/heartjnl-2015-309110

[5] Draulans-Noe, H.A., Wenink, A.C. and Quaegebeur, J. (1990) Single Papillary Muscle ("Parachute Valve") and Double-Orifice Left Ventricle in Atrioventricular Septal Defect Convergence of chordal Attachment: Surgical Anatomy and Results of Surgery. Pediatric Cardiology, 11, 29-35. https://doi.org/10.1007/BF02239544

[6] Nijenhuis, V.J., Sanchis, L., van der Heyden, J.A.S., Klein, P., Rensing, B.J.W.M., Latib, A., Maisano, F., Ten Berg, J.M., Agostoni, P. and Swaans, M.J. (2017) The Last Frontier: Transcatheter Devices for Percutaneous or Minimally Invasive Treatment of Chronic Heart Failure. Netherlands Heart Journal. [Epub Ahead of Print] https://doi.org/10.1007/s12471-017-1018-8 